\title{
El acercamiento a las fuentes de la Historia como método de desarrollo de las competencias necesarias para la escritura académica
}

\section{Francisco J. Molina de la Torre ${ }^{a}$ y Sofía Pizarro Riñón}

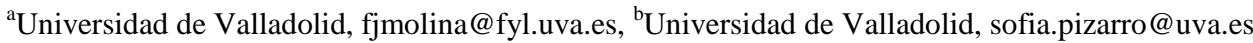

\begin{abstract}
The task of the historian is to create a narrative out of a number of very different historical sources. Writing, therefore, is an essential tool for all students and, actually, more than a tool: writing is learning. However, many of today's university students must be taught how to write at the academic level. The present paper explores an experience held at the University of Valladolid, in which students were given the opportunity to have direct contact with historical sources and to write a tutorized online essay about such sources.
\end{abstract}

\section{Keywords:}

History, historical sources, writing, collaborative learning, online tutorization.

\begin{abstract}
Resumen
La tarea del historiador es elaborar un relato a partir de una serie de fuentes escritas muy diversas, por lo que escribir se convierte en una herramienta imprescindible para todos los estudiantes. De hecho, es más que una herramienta, pues al escribir se aprende. Sin embargo, hoy en día es preciso enseñar a muchos de nuestros estudiantes universitarios a escribir un texto académico. Este trabajo analiza una experiencia desarrollada en la Universidad de Valladolid en la que los estudiantes tuvieron la oportunidad de entrar en contacto directo con las fuentes escritas y elaborar un trabajo tutorizado online acerca de dichas fuentes.
\end{abstract}

Palabras clave: Historia, fuentes históricas, escribir, aprendizaje colaborativo, tutorización online.

\section{Introducción}

La correcta expresión, tanto oral como escrita, constituye una de las más importantes competencias en la mayor parte de los perfiles profesionales, tanto en el campo de las humanidades y las ciencias sociales como en el de las ciencias de la salud y otras ciencias. Sin duda, en el caso de la titulación del Grado en Historia se erige como un pilar fundamental, de tal modo que, por ejemplo, la Universidad de Valladolid lo contempla tanto entre los objetivos generales del grado como entre las competencias generales, específicas y trasversales. Para lograrlo, en la exposición de los objetivos y competencias, 
se recoge que "la realización de trabajos escritos a lo largo de las diferentes asignaturas y, en especial, del Trabajo de Fin de Grado, pretenden desarrollar esta competencia” (UVA, Objetivos y competencias).

Además, desde la segunda mitad del siglo XX, a partir de los estudios de Brenner, Luria y, en particular, Vygotsky, se ha puesto de manifiesto que la escritura es un elemento clave en el proceso mismo de aprendizaje: no solo aprendemos a escribir, sino que escribiendo aprendemos. De este modo, la escritura se convierte no solo en una meta a alcanzar, sino también en un instrumento fundamental en todo proceso de enseñanza-aprendizaje.

Sin embargo, son frecuentes las quejas entre los docentes universitarios a causa del escaso dominio del registro escrito por parte de los alumnos, algo que, de todos modos, no ha sido objeto de estudio detenido en el ámbito español, a diferencia de lo que sucede en otros países hispanoparlantes (Gallego Ortega y Mendías Cuadros, 2012: 48). En cualquier caso, el panorama que se describe sobre la escritura entre los universitarios colombianos (Andrade Calderón, 2009: 326-327), caracterizado por la desmotivación, las faltas de ortografía, la mala estructuración del texto, el desconocimiento del tema sobre el que se escribe, entre otras cosas, no dista demasiado de lo que encontramos en nuestras aulas. Así pues, la realidad a la que nos enfrentamos se caracteriza, por un lado, por un alumnado que en buena medida ignora aspectos básicos de la escritura y, por otro lado, docentes que, siguiendo lo postulado en los programas, exigen la redacción de distintos tipos de trabajos escritos -incluido el Trabajo de Fin de Grado- pero que, considerando que todo alumno universitario ya debería dominar el registro escrito, se limita a evaluar sin más dichos trabajos.

Por otra parte, en el campo de la Historia, no podemos olvidar la trascendencia que las fuentes escritas tienen para la misma. Aunque no puede escribirse historia sin recurrir a los testimonios llegados a nosotros de los distintos periodos históricos, hay ocasiones en que el aprendizaje de la historia se convierte en la memorización de unos apuntes o unos manuales elaborados por el profesor o por un grupo de expertos en la materia, de tal manera que el alumno no tiene acceso directo a la documentación original que ha servido a los historiadores para construir su relato histórico.

Un elemento adicional que ha de tenerse en cuenta es la compleja implantación de las TICs en la enseñanza y aprendizaje de la historia. Si bien es cierto que en la actualidad hay numerosas posibilidades, también lo es que el número de docentes interesados por ellas es bastante reducido. Muestra clara del desinterés de los historiadores por la innovación docente es el número de comunicaciones recogidas en las actas de las ediciones anteriores de este Congreso vinculadas a dicho campo de estudio (no hemos sido capaces de identificar ningún trabajo propiamente histórico, aun cuando algunos versan sobre historia del arte, historia de la música, etc.).

Teniendo en cuenta todo lo anterior, un grupo de docentes de Historia de la Universidad de Valladolid, junto con algunos docentes de enseñanzas medias, planteamos un Proyecto de Innovación Docente con el título de "Las fuentes de la historia. Un binomio en la construcción de aprendizajes”. Con él pretendíamos subsanar algunas de las deficiencias

(cc) BY-NC-ND 2018, Universitat Politècnica de València

Congreso IN-RED (2018) 
observadas en la formación de los alumnos de Historia, al mismo tiempo que mejorar sus capacidades como docentes y avanzar en la reflexión sobre la auténtica mejora de la universidad. Dentro del abanico de actividades diseñadas y desarrolladas en el seno del proyecto, los llamados seminarios "Mil Historias" tenían por objeto acercar a los estudiantes a las fuentes primarias, al mismo tiempo que acompañarlos personalmente en el proceso de redacción de un trabajo escrito empleando algunos recursos de la nube.

El presente trabajo, por consiguiente, recoge la experiencia de los seminarios "Mil Historias”, desde su concepción hasta su evaluación por parte de los participantes y de los miembros del proyecto de innovación docente. No obstante, la mejor valoración tendrá lugar cuando, al año próximo, los estudiantes se enfrenten a la tarea de redactar su Trabajo de Fin de Grado y tengan la experiencia previa de haber redactado un trabajo tutorizado, con recursos 2.0 y en cuya elaboración han encontrado la colaboración, no solo de los docentes implicados, sino de otros alumnos participantes en el proyecto.

\section{Objetivos}

1. Acercar las fuentes escritas de la historia a los alumnos tanto a través de los recursos digitales como facilitando el acceso a los archivos de la ciudad de Valladolid.

2. Proporcionar a los alumnos herramientas para el análisis de las fuentes históricas y la elaboración de un discurso histórico a partir de las mismas.

3. Mostrar, por medio de ejemplos, cómo se elabora la historia a partir de diversas fuentes.

4. Ofrecer la posibilidad de realizar un trabajo tutorizado en el que se puedan poner en práctica las competencias a desarrollar.

5.Promover el uso de herramientas 2.0 para facilitar un aprendizaje colaborativo entre profesores y alumnos que enriquezca el resultado de los trabajos realizados.

\section{Desarrollo}

\subsection{Planificación}

La idea de crear un proyecto de innovación en el Grado en Historia de la Universidad de Valladolid surgió a raíz de la Convocatoria de Proyectos de Innovación Docente 2017-2018 publicada el 3 de julio, para "fomentar y apoyar el diseño y desarrollo de proyectos de innovación educativa que tienen como objetivo final mejorar la capacitación y cualificación de nuestros estudiantes” (UVA, Convocatoria).

Ante este reto, algunos docentes preocupados por la escasa capacidad de redacción que demuestran los alumnos en las pruebas de evaluación de las distintas asignaturas, así como por la falta de herramientas de que disponen a la hora de enfrentarse a la elaboración del TFG, crearon un equipo de profesores de diferentes áreas para trabajar cooperativamente en el desarrollo de la innovación. 
En el curso de una reunión preparatoria en la que se analizaron los problemas existentes que se planteaban resolver, las posibilidades que proporcionaba la estructura del grado y los recursos humanos con los que se contaban, surgió el proyecto ya mencionado de los seminarios "Mil Historias".

Se trataba de una actividad de carácter extracurricular y de naturaleza voluntaria dirigido fundamentalmente a los alumnos de $2 .^{\circ}$ y $3 .^{\circ}$ de grado. El público se eligió al considerar que los estudiantes, en estos cursos, ya cuentan con algunos rudimentos propios de la Historia, y disponen además de cierto bagaje y herramientas, como la paleografía, que les permiten acceder a las fuentes escritas de distintas épocas con cierta comprensión del contexto histórico en el que se enmarcan. Por otro lado, son cursos previos a la elaboración del TFG, en los que que se pueden ir asimilando los conocimientos que ayudarán al alumno a la realización de este trabajo.

A través de esta actividad se pretendía, en la línea del modelo educativo planteado por el EEES para el Grado en Historia (ANECA, 2004), el desarrollo de competencias por medio de una construcción activa y personal del aprendizaje, entendidas las competencias como la "combinación de conocimientos, capacidades, habilidades, actitudes y valores que se requieren para la comprensión y transformación de una realidad compleja” (Mateo, 2006; citado por Climent, 2010: 92). Entre otros aspectos, interesaba fomentar una mayor capacidad crítica, una autonomía en el aprendizaje y una curiosidad por conocer, junto con la ya mencionada capacidad de comunicación oral y escrita, lo que se ha tratado de llevar a cabo por medio del acercamiento a las fuentes escritas primarias.

El desarrollo de los seminarios se planteó con dos fases, una primera en la que se mostraría a los alumnos las posibilidades de estudio que presenta una sola fuente, y una segunda en la que los alumnos se convertirían en los protagonistas elaborando su propio trabajo a partir de un documento histórico.

\subsection{La primera fase de los seminarios "Mil Historias"}

La primera fase consistió en la elaboración de unos seminarios denominados "Mil Historias”. El título hacía referencia a los diferentes relatos que se pueden extraer a través de una fuente histórica y tenían como objetivo mostrar a los alumnos cómo debe ser la actitud del historiador ante los testimonios gráficos para hacer "hablar" a las fuentes.

El formato elegido para estas exposiciones fue el de seminario, es decir, una reunión especializada en la que se desarrolla un estudio profundo sobre una materia concreta, en la que además del docente, los alumnos también participan por medio de un aprendizaje activo interactuando con el guía (Monografías, 2018). Este formato ha sido empleado con éxito en otras experiencias de innovación docente (Mariles, 2012; Saiz y Susinos, 2017). De esta forma, los alumnos pudieron exponer sus dudas acerca del tema tratado, intercambiar impresiones y aportar nuevas ideas.

Los seminarios se desarrollaron en tres sesiones. La primera de ellas, titulada "La inscripción bajo la escalera del edificio histórico de la Universidad de Valladolid”, versó

(cc) BY-NC-ND 2018, Universitat Politècnica de València

Congreso IN-RED (2018) 
sobre una fuente epigráfica de época contemporánea. En este seminario se reflexionó sobre el carácter comunicativo de las inscripciones, el mensaje que pretenden comunicar, las personas y oficios implicados en su realización, descubriendo las múltiples investigaciones que se pueden realizar a partir de ellas.

En el segundo seminario se mostraron las posibilidades de investigación que ofrece un documento, en concreto, el sermón pronunciado en 1511 por Antonio de Montesinos, fraile dominico, en la isla de La Española sobre la defensa de la libertad e integridad de las poblaciones indígenas americanas. A través de él se presentaron las posibilidades de estudio para la conquista de América, en sus múltiples facetas, organización administrativa, encomienda, población indiana y las consecuencias que tuvo como punto de partida en el movimiento de defensa de los indios.

Por último, tuvo lugar un seminario en el que se estudió un libro y las diferentes vertientes de interpretación del mismo a partir del códice, o códices, de Las Partidas de Alfonso X el Sabio. En él se hizo hincapié en la necesidad de la interdisciplinariedad a la hora de elaborar un estudio como forma de enriquecer los resultados obtenidos y se puso en valor una fuente jurídica para el estudio de diversas facetas de la historia, además del derecho, como la sociedad y la economía.

La participación en los seminarios contó con la presencia entre 8 y 12 alumnos de $2 .^{\circ}$ y $3 .^{\circ}$ curso del Grado en Historia. Aunque pueda parecer una asistencia reducida, hay que tener en cuenta que el número de los alumnos matriculados por curso suele oscilar entre 50-60 estudiantes, por lo que la asistencia sería de algo más de un $10 \%$. De todas formas, esta baja implicación en el proyecto es una muestra más de la desmotivación que existe entre los universitarios.

\subsection{Los seminarios "guía"}

Una vez celebrados los tres seminarios "Mil Historias", comenzaba la segunda fase, la cual daba sentido al proyecto, pues era esta en la que los estudiantes se implicaban de forma directa. Para orientar a los participantes, organizamos dos seminarios "guía", uno al comienzo de esta fase y otro transcurrido un mes.

El primero de dichos seminarios tenía por objeto conocer el número real de participantes en esta fase del proyecto, elegir el tema sobre el que cada alumno deseaba trabajar y dar unas orientaciones generales sobre la redacción de los trabajos (vinculándolo con la redacción futura del TFG) y el uso de Google Drive.

Al final, fueron tan solo cinco los alumnos que decidieron voluntariamente participar en la segunda fase de los seminarios. Ello, pese a que parezca un número en exceso reducido, nos ofrecía la oportunidad de trabajar de una manera mucho más personalizada con los participantes. Así pues, ello nos permitía incidir en aquellos aspectos que han dado en llamarse "aprendizajes colaterales" (González Geraldo, 2014: 62-66), es decir, aquellos aspectos de la educación que no están planificados pero que son bien percibidos por los alumnos, pues "es posible que los alumnos aprendan más de sus profesores que de las 
propias materias” (González Geraldo et al., 2012: 198). En este sentido, además de la relación con los estudiantes en el marco de los seminarios (y, por consiguiente, fuera del ámbito académico estricto, permitiendo así una mayor cercanía y espontaneidad), el hecho de que algunos optaran por trabajar sobre fuentes de los archivos de la ciudad nos dio ocasión de acompañarles a los archivos, guiarles en la solicitud de la documentación, hablar con ellos sobre sus expectativas y dificultades, conocer su opinión sobre el Grado, etc.

En el segundo de los seminarios "guía” buscábamos poner en común y resolver de forma colaborativa algunas de las dificultades que los participantes en los seminarios se hubiesen encontrado.

Dichas dificultades iban a ser fundamentalmente de tres tipos: unas estaban relacionadas con el entorno tecnológico, pues, a pesar de que Google Drive no parece suponer ningún reto, su uso en nuestros ámbitos educativos es bastante reducido; otras complicaciones derivaban de la propia naturaleza de la fuente elegida para hacer el trabajo -bien por su extensión o bien por la dificultad de desentrañar el contenido de la misma (recordemos que la escritura castellana de los siglos XVI y XVII es, en muchos casos, difícil de comprender)-; y, finalmente, otra serie de dificultades estaban vinculadas al proceso mismo de redacción, desde la organización de las ideas, pasando por problemas de sintaxis y signos de puntuación, hasta la forma de citar.

Además de comentar las dificultades y de proponer algunas pautas generales para continuar con los trabajos, se incidió en la importancia de la colaboración de todos en la redacción de los textos. Para ello se comentó el artículo de Castelló, González e Iñesta (2010), que tenían disponible en un repositorio del Google Site en el que trabajábamos. En ese sentido, se advirtió que los comentarios ofrecidos a los demás debían ser constructivos, siendo admisibles correcciones de estilo y de ortografía, pero insistiendo en la importancia sobre todo de abrir nuevas perspectivas desde la experiencia personal.

Por último, en dicho seminario planteamos la posibilidad de que al final los trabajos se expusieran ante los demás participantes del proyecto. Era un tema que, dado que el proyecto estaba principalmente orientado hacia la expresión escrita, habíamos dejado en suspenso para conocer la opinión de los estudiantes. En definitiva, a la luz del debate que se suscitó, optamos por celebrar uno o dos seminarios de exposición, de modo que la experiencia no solo sirviese para trabajar en la comunicación escrita, sino en la oral, pues también esta es un elemento fundamental en la formación de futuros historiadores y docentes de historia.

\subsection{El trabajo online tutorizado}

Tras el primer seminario "guía” se dio paso a la elaboración de los trabajos de los estudiantes. En el Google Site se tenía acceso tanto al documento en el que elaborar personalmente el trabajo como a los documentos de los otros participantes, donde se podía intervenir libremente.

(cc) BY-NC-ND 2018, Universitat Politècnica de València

Congreso IN-RED (2018) 
A pesar de las dificultades iniciales (fundamentalmente derivadas de las diferencias entre el programa Word, con el que los participantes estaban más familiarizados, y el editor de texto de Google), en un plazo relativamente breve -dos o tres semanas- los participantes habían comenzado a redactar sus textos.

Dado que el número de participantes, como hemos indicado, no era muy elevado, la labor de tutorización la realizamos los dos autores del presente trabajo, recurriendo en algunos momentos a otros miembros del Proyecto de Innovación Docente. Dicha tutorización consistió sobre todo en hacer comentarios en el documento de Google relativos a muy diversos aspectos, tanto a nivel ortográfico y sintáctico como de estructuración del relato histórico. Procuramos respetar el estilo propio de cada estudiante, si bien hicimos sugerencias o comentarios que creíamos que les serviría primero para mejorar el texto que estaban preparando, pero también para futuras redacciones (en particular para el TFG).

Dado que observamos que la participación de los alumnos en los trabajos de los demás era bastante escasa, creímos conveniente, según hemos indicado en el apartado interior, que en el segundo seminario "guía" se tratase de esta cuestión. Nos pareció que el mencionado trabajo de Castelló, González e Iñesta, pese a que remite a los trabajos conducentes al doctorado, les resultaría esclarecedor, pues, como las autoras manifiestan de forma rotunda, "la revisión colaborativa mejora los textos finales" (2010: 534). A partir de ese seminario "guía" aumentó el número de intervenciones de los estudiantes en los textos de sus compañeros, produciéndose en algunos casos un intercambio de ideas realmente enriquecedor.

Al cabo de dos meses dimos por cerrado la redacción de los textos, que probablemente sirvan como base para futuros proyectos con nuevos estudiantes que quieran participar en los seminarios.

\subsection{Los seminarios de exposición}

Como hemos indicado más arriba, en el proyecto original de los seminarios "Mil Historias" no contemplábamos de forma definitiva la exposición de los trabajos realizados. El hecho de que el número de participantes fuera reducido, así como el interés que manifestaron cuando les propusimos la posibilidad, nos llevó a organizar los "seminarios de exposición", en los que brindamos a los participantes la oportunidad de exponer sus trabajos ante sus compañeros (la exposición estaba abierta a otros estudiantes que no habían participado en los seminarios).

A fin de disponer de tiempo para la exposición y la revisión de la misma, optamos por celebrar dos sesiones. En la primera expusieron su trabajo dos estudiantes y en la segunda otros dos (un participante no pudo exponerlo por diversas razones). En cada seminario, el procedimiento que se siguió fue el siguiente. Comenzábamos con la exposición por parte del estudiante, para la cual tenía un tiempo asignado de quince minutos. La mayoría se sirvió de un apoyo visual para la misma (fundamentalmente PowerPoint, aunque en un caso se empleó Prezi). La exposición era grabada por un miembro del Proyecto. Al acabar la exposición, se abría un tiempo de reflexiones generales en el que los presentes podían hacer 
sus comentarios acerca de la exposición -en este caso, advertimos que no nos interesaba tanto el contenido de la exposición cuanto la forma de la misma-.

Después de las dos exposiciones de los alumnos y de los comentarios generales, una vez que habían salido quienes no formaban parte de los seminarios, ofrecíamos la posibilidad de hacer un análisis más detenido de la exposición revisando el vídeo, a fin de identificar tanto las fortalezas como las debilidades de la misma y poder mejorar en el futuro. Con quienes accedieron a revisarlo, descubrimos algunas de las deficiencias más habituales que se observan en este tipo de presentaciones: velocidad excesiva en la dicción provocada por el nerviosismo, abuso de algunas muletillas, tono monótono, uso de demasiado texto en la presentación PowerPoint, etc.

\section{Resultados}

Los resultados que exponemos a continuación derivan fundamentalmente de las impresiones y datos que los miembros del proyecto hemos ido recabando mediante el diálogo con los participantes y el debate entre nosotros. Asimismo, en algunos casos (para los resultados 4 y 5) hemos aprovechado las encuestas que vía Google Forms han ido realizando los participantes en los seminarios para conocer el parecer de los participantes de una forma más formal.

\subsection{Los estudiantes entran en contacto directo con las fuentes de la historia.}

Uno de los grandes objetivos que nos planteábamos era facilitar a los participantes en el proyecto el contacto directo con las fuentes. Ello se ha logrado no solo mediante la participación en los seminarios de la primera fase (cuando se presentaba una fuente y las "mil historias" que pueden escribirse a partir de la misma), sino ante todo con los trabajos tutorizados. En este caso, los propios estudiantes han elegido la fuente sobre la que deseaban trabajar, de forma que el elenco de fuentes ha sido muy variado, tanto en su tipología como en su cronología, e incluso se ha trabajado sobre fuentes inéditas conservadas en el Seminario Diocesano, que podrían ser material para un futuro TFG.

\subsection{Los estudiantes escriben y, consiguientemente, aprenden.}

El mero hecho de ponerse a escribir constituye un elemento fundamental en el proceso de aprendizaje de los estudiantes. Además, advierten cómo los contenidos se fijan mucho más al dedicar tiempo a poner por escrito las ideas, pues exige un ejercicio de reflexión, de organización y de expresión que sin duda favorece la asimilación del tema sobre el que están trabajando.

\subsection{Los estudiantes advierten cómo la tutorización de los trabajos permite una mejora sustancial de los mismos.}

La tutorización de los trabajos ha posibilitado, en primer lugar, la identificación de las principales deficiencias de los alumnos a la hora de enfrentarse a la redacción de un texto. Ello ha contribuido no solo a que los alumnos adviertan en qué aspectos han de mejorar en su trabajo, sino también a que nosotros, como docentes, especialmente como tutores de 
TFGs, descubramos cuáles son los errores más frecuentes y podamos orientar mejor a los estudiantes. En segundo lugar, la tutorización mediante Google Drive ha permitido que los participantes se habituasen a trabajar en un entorno virtual, algo que, como señalamos anteriormente, no es excesivamente habitual en el mundo de la historia.

\subsection{Los estudiantes aprenden que el trabajo en colaboración con sus compañeros es un instrumento muy positivo para enriquecer los textos que escriben.}

El uso de Google Drive facilitó el intercambio de comentarios y sugerencias entre los participantes, si bien fue necesario insistir en ello para que los alumnos realmente interviniesen en el trabajo de los demás. En cualquier caso, además, como aparece en algunas de las evaluaciones de los alumnos, estos tienen interés en "preguntar dudas o vuestra opinión en caso de necesidad", y las respuestas brindadas no solo por los docentes, sino también por sus compañeros son generalmente bien valoradas.

\subsection{Los estudiantes muestran interés cuando se les ofrecen actividades orientadas a completar y mejorar su formación.}

Ciertamente se trata de algo que no puede aplicarse a la mayor parte del alumnado, pero sí a un porcentaje limitado (10-15\%), que participa en actividades académicas extraordinarias aun cuando no les reporten ningún tipo de beneficio en forma de créditos. Además, la participación no se limita a acudir a una charla y escuchar, sino que se muestran activos (evaluando las actividades, ofreciendo sugerencias para futuros proyectos y sobre todo redactando un trabajo tutorizado). Por ejemplo, en el caso de los seminarios de la primera fase, entre las sugerencias se planteaba "la posibilidad de enviar textos antes del seminario para prepararlos en casa y así poder comentarlos con el conferenciante”.

\section{Conclusiones}

La participación en el proyecto, al ser totalmente voluntaria, no ha sido muy elevada, como ya hemos mencionado, sin embargo podemos decir que para los alumnos que se han involucrado hasta el final ha sido una experiencia muy satisfactoria, cumpliéndose los objetivos propuestos inicialmente.

El trabajo con las fuentes escritas, sin las que no podríamos elaborar la historia, han mostrado a los participantes el verdadero significado del Grado que estudian, que no consiste únicamente en memorizar una lista interminable de hechos o personajes, sino en desarrollar la capacidad de comprender el pasado, profundizando en los hechos acaecidos, para entender el presente y mirar hacia el futuro. De esta forma han experimentado el papel que tiene el historiador a la hora de extraer la información que nos proporcionan las fuentes y generar nuevos relatos, estimulando en ellos la inquietud y curiosidad necesarias para el desarrollo de la ciencia.

El estudio de la documentación les ha llevado a adquirir algunas competencias transversales y específicas del grado de Historia, como la capacidad de análisis y la capacidad de relacionar lo estudiado en las distintas asignaturas con la investigación realizada. 
Los resultados del proceso de enseñanza-aprendizaje se han visto incrementados gracias al trabajo colaborativo, ya que en en el transcurso de la elaboración de los trabajos han podido participar aportando sugerencias, indicaciones, diversos puntos de vista, tanto los profesores vinculados al proyecto como los demás alumnos, que han enriquecido el resultado final. Esta colaboración del grupo, aunque el peso fundamental de la redacción era personal, ha sido factible gracias a las posibilidades que nos brindan las TIC, las cuales permiten hacer contribuciones sin necesidad de encontrarse reunidos, haciendo de esta forma más dinámicas las intervenciones. De todas formas, tampoco podemos olvidar los seminarios como otra herramienta, más tradicional, que ha permitido fomentar la colaboración.

Por lo tanto, podemos concluir que el trabajo colaborativo ha optimizado los resultados obtenidos favoreciendo el aprendizaje significativo y que las herramientas 2.0 han permitido, a su vez, llevar a cabo un trabajo más colaborativo y dinámico.

El proyecto ha concluido, por consiguiente, con el deseo de perdurar en años consecutivos ampliando su radio de acción a un número más amplio de estudiantes y con la esperanza de que las competencias adquiridas por los alumnos participantes tengan sus frutos en la elaboración de los futuros TFG, elevando el nivel de resultados de los mismos.

\section{Referencias}

ANDRAde CALderón, M. C. (2009). "La escritura y los universitarios” en Universitas humanistica, n. ${ }^{\circ}$ 68, julio-diciembre, pp. 297-340.

ANECA (2004). Libro Blanco: Título de Grado en Historia. $<$ http://www.aneca.es/var/media/150448/libroblanco_jun05_historia.pdf $>$ [Consulta: 18 de marzo de 2018].

CAstelló, M., GonzÁlez, D., IÑEsta, A. (2010). “La regulación de la escritura académica en el doctorado: el impacto de la revisión colaborativa en los textos” en Revista española de pedagogía, año LXVIII, n. ${ }^{\circ}$ 247, septiembre-diciembre, pp. 521-537.

Climent Bonilla, J. B. (2010). "Reflexiones sobre la Educación Basada en Competencias" en Revista Complutense de Educación, vol. 21, n. ${ }^{\circ}$ 1, pp. 91-106.

Gallego Ortega, J. L., y Mendías Cuadros, A. M. (2012). “¿Qué saben los estudiantes universitarios sobre la planificación de tareas de escritura? Un estudio de caso” en Revista de Investigación en Educación, $\quad$ n. ${ }^{\circ} \quad 10 \quad$ (2), $\quad$ pp. $47-61$. $<$ https://dialnet.unirioja.es/descarga/articulo/4732994.pdf> [Consulta: 15 de marzo de 2018].

GONZÁlez GERAldo, J. L. (2014). Hacia una universidad más humana. ¿Es superior la educación superior. Madrid: Biblioteca Nueva.

GonZÁLEZ GERALDO, J. L. et al. (2012). “Aprendizajes colaterales: límites y retos del aprendizaje por competencias” en ENSAYOS. Revista de la Facultad de Educación de Albacete, n. ${ }^{\circ}$ 29, 189-202. $<$ https://www.revista.uclm.es/index.php/ensayos/article/view/247> [Consulta: 20 de marzo de 2018].

MARILES Mora, S. (2012). "El seminario como fuente de aprendizaje cooperativo en el desarrollo de las ciencias biológicas” en Horizontes pedagógicos, vol. 14, n. ํ 1, pp. 141-155.

(cc) EY-NC-ND 2018, Universitat Politècnica de València

Congreso IN-RED (2018) 
MonografíAs, El Seminario. $\quad<$ http://www.monografias.com/trabajos93/seminariodiapositivas/seminario-diapositivas.shtml> [Consulta: 18 de marzo de 2018].

Saiz Linares, Á. y Susinos RadA, T. (2017). "Los seminarios colaborativos en un practicum reflexivo de maestros. Análisis de una experiencia en la Universidad de Cantabria (España)” en Perspectiva Educacional. Formación de Profesores, vol. 56 (3), pp. 3-24. $<$ file:///C:/Users/Sof\%C3\%ADa/Downloads/DialnetLosSeminariosColaborativosEnUnPracticumReflexivoDe-6153547.pdf> [Consulta: 18 de marzo de 2018].

UnIVERSIDAD DE VALlADOlid. Objetivos y competencias del Grado en Historia. $<$ http://www.uva.es/export/sites/uva/2.docencia/2.01.grados/2.01.02.ofertaformativagrados/_documen tos/historia_competencias.pdf $>$ [Consulta: 15 de marzo de 2018].

Universidad DE VAlLADOLID. Convocatoria de Proyectos de Innovación Docente. $<$ http://www.uva.es/export/sites/uva/7.comunidaduniversitaria/7.01.pdi/7.01.04.innovacioneducativa/ _documentos/Sellado_ConvocatoriaPID17-18.pdf> [Consulta: 16 de marzo de 2018]. 